JMB AP

\title{
Molecular Architecture of the Undecameric Rotor of a Bacterial $\mathrm{Na}^{+}$-ATP Synthase
}

\author{
Janet Vonck ${ }^{1 *}$, Tassilo Krug von Nidda ${ }^{1}$, Thomas Meier ${ }^{2}$ \\ Ulrich Matthey ${ }^{2}$, Deryck J. Mills ${ }^{1}$, Werner Kühlbrandt ${ }^{1}$ and \\ Peter Dimroth ${ }^{2}$
}

\author{
${ }^{1}$ Max-Planck-Institute of \\ Biophysics \\ Heinrich-Hoffmann-Str. 7 \\ D-60528 Frankfurt, Germany \\ ${ }^{2}$ Institut für Mikrobiologie der \\ ETH Zürich \\ Schmelzbergstrasse 7, CH-8092 \\ Zurich, Switzerland
}

\begin{abstract}
The sodium ion-translocating $\mathrm{F}_{1} \mathrm{~F}_{0}$ ATP synthase from the bacterium Ilyobacter tartaricus contains a remarkably stable rotor ring composed of 11 c subunits. The rotor ring was isolated, crystallised in two dimensions and analysed by electron cryo-microscopy. Here, we present an $\alpha$-carbon model of the c-subunit ring. Each monomeric c subunit of 89 amino acid residues folds into a helical hairpin consisting of two membrane-spanning helices and a cytoplasmic loop. The $11 \mathrm{~N}$-terminal helices are closely spaced within an inner ring surrounding a cavity of $\sim 17 \AA(1.7 \mathrm{~nm})$. The tight helix packing leaves no space for side-chains and is accounted for by a highly conserved motif of four glycine residues in the inner, N-terminal helix. Each inner helix is connected by a clearly visible loop to an outer C-terminal helix. The outer helix has a kink near the position of the ion-binding site residue Glu65 in the centre of the membrane and another kink near the $C$ terminus. Two helices from the outer ring and one from the inner ring form the ion-binding site in the middle of the membrane and a potential access channel from the binding site to the cytoplasmic surface. Three possible inter-subunit ion-bridges are likely to account for the remarkable temperature stability of I.tartaricus c-rings compared to those of other organisms.
\end{abstract}

(C) 2002 Elsevier Science Ltd. All rights reserved

Keywords: electron crystallography; 2-D crystals; $\mathrm{F}_{1} \mathrm{~F}_{0}$ ATP synthase; c-ring; $\mathrm{Na}^{+}$translocation or 14 for the ATP synthases from yeast mitochondria, ${ }^{4}$ from the bacterium Ilyobacter tartaricus ${ }^{5}$ or from spinach chloroplasts, ${ }^{6}$ respectively. In bacteria, the $\mathrm{F}_{0}$ part consists of two additional subunit species $a$ and $b_{2}$, which are connected laterally to the c-ring. ${ }^{7-10}$ The major part of the two $b$ subunits is $\alpha$-helical, comprising the peripheral stalk that connects subunit a to a complex of subunits $\alpha$ and $\delta .{ }^{11,12}$

The ATP synthase is the smallest known rotary motor. Several pieces of experimental evidence indicate that ATP hydrolysis elicits the rotation of the rotor subunits $\gamma, \varepsilon$ and $\mathrm{c}_{10-14}$ against the stator subunits $\alpha_{3} \beta_{3} \delta \mathrm{ab}_{2} \cdot{ }^{13-15}$ Most current models for ATP synthesis imply that the flux of $\mathrm{H}^{+}$or $\mathrm{Na}^{+}$across $\mathrm{F}_{0}$ induces rotary movement of the rotor versus the stator. This rotation promotes conformational changes of the $\beta$ subunits that are instrumental for catalysing ATP synthesis and releasing tightly bound ATP from the catalytic sites. ${ }^{1,16}$

Details of the torque-generating mechanism are unknown, mainly because structural information

Abbreviations used: AFM, atomic force microscopy.

E-mail address of the corresponding author: vonck@mpibp-frankfurt.mpg.de 
Table 1. Crystallographic data

\begin{tabular}{ll}
\hline Plane group & $p 1$ \\
Unit cell parameters & $91.7,89.7$ \\
$a, b(\AA)$ & 114.9 \\
$\gamma($ deg.) & $55\left(10 \text { at } 0^{\circ}, 6 \text { at } 20^{\circ}, 26 \text { at } 30^{\circ}, 13 \text { at } 45^{\circ}\right)^{\mathbf{a}}$ \\
Number of images & 15,242 \\
Number of observations & 19.5 \\
Overall weighted phase error & \\
Effective resolution $(\AA)$ & 4 \\
In-plane & $\sim 15^{\mathbf{b}}$ \\
Vertical &
\end{tabular}

a Untilted images were merged to $4 \AA$, tilted images to $6 \AA$ resolution.

b From the point spread function. ${ }^{53}$

on the $\mathrm{F}_{0}$ part of the molecule is limited. So far, most structural studies have been performed with subunit c. Atomic force microscopy (AFM) images and projection maps obtained by electron microscopy have been described for the undecameric rotor ring of I. tartaricus ${ }^{5}$ and for the $\mathrm{c}_{14}$ oligomer from spinach chloroplasts. ${ }^{6}$ Furthermore, a $3.9 \AA$ X-ray structure is available for the $F_{1} c_{10}$ complex from yeast. ${ }^{4}$ Monomeric subunit $\mathrm{c}$ from Escherichia coli, studied by NMR in organic solvent, was found to fold as a helical hairpin, ${ }^{17}$ confirming previous labelling experiments with the membrane-bound complex. ${ }^{18}$ In contrast to subunit c of E. coli, that of Propionigenium modestum did not fold into a stable 3-D structure in detergent or in organic solvent, possibly because the structure must be stabilised by intersubunit contacts. ${ }^{19,20}$

The mechanism of $\mathrm{H}^{+}$or $\mathrm{Na}^{+}$translocation across $F_{0}$ involves binding of the coupling ions to the $c$ subunit binding site. ${ }^{21}$ This is a conserved acidic residue in the $\mathrm{C}$-terminal helix in the case of $\mathrm{H}^{+}$-translocating ATP synthases and includes Gln32 on the N-terminal helix and Glu65 and

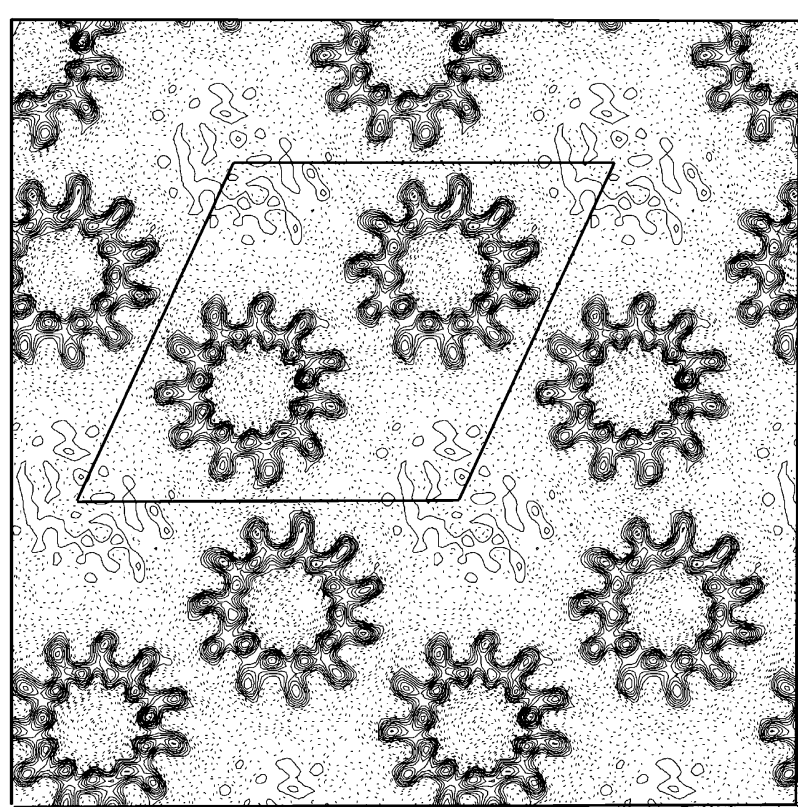

Figure 1. Projection map of a c-ring crystal image at $4 \AA$ resolution. One unit cell is outlined.
Ser66 on the C-terminal helix in the $\mathrm{Na}^{+}$-translocating ATP synthases from P.modestum or I. tartaricus. $^{22}$ Despite extensive biochemical evidence for direct access from the $\mathrm{Na}^{+}$-binding site to the cytoplasmic surface, ${ }^{23,24}$ recent crosslinking data have shown that the site is in contact with the fatty acid side-chains of phospholipids and therefore in the centre of the membrane. ${ }^{25}$

An elegant solution to this dilemma becomes apparent from our present work, where we present an electron crystallographic model of the $c_{11}$ ring from I. tartaricus. We can locate the ion-binding site near the middle of the membrane where ligands are contributed from one inner helix and two outer helices, and a putative access channel between the binding site and the cytoplasm can be identified.

\section{Results and Discussion}

\section{The 3-D map of the c-ring}

We have shown that the $\mathrm{Na}^{+}$-translocating $\mathrm{F}_{1} \mathrm{~F}_{0}$ ATP synthase of I. tartaricus contains a c subunit rotor ring of unusual stability that resists boiling in SDS solution for five minutes. ${ }^{26}$ Analysis of the $\mathrm{F}_{1} \mathrm{~F}_{0}$ ATP synthase or the isolated rotor ring by SDS-PAGE showed the same mobility for the $c$ oligomer, indicating that the number of c subunits in the oligomer did not change during the isolation procedure. A native-like structure of the isolated c oligomer is indicated by reconstitution experiments: upon combining the $c$ oligomer with subunits $a$ and $b$ and with the $F_{1}$ headpiece functional, $\mathrm{Na}^{+}$-coupled ATP synthase was recovered. ${ }^{27}$ Furthermore, the modification of glutamate 65 of subunit c by dicyclohexylcarbodiimide (DCCD) in the $\mathrm{F}_{1} \mathrm{~F}_{0}$ complex or in the isolated $\mathrm{C}$ subunit oligomer was indistinguishable kinetically (unpublished results). Hence, we are confident that the isolated $c$ ring of I. tartaricus retains its native structure.

The isolated c rings were crystallised in two dimensions and projection maps of the 2-D crystals showed that the rings are composed of 11 subunits. ${ }^{5}$ We have improved the quality of the 2-D crystals, which grew up to $2 \mu \mathrm{m}$ in size. 

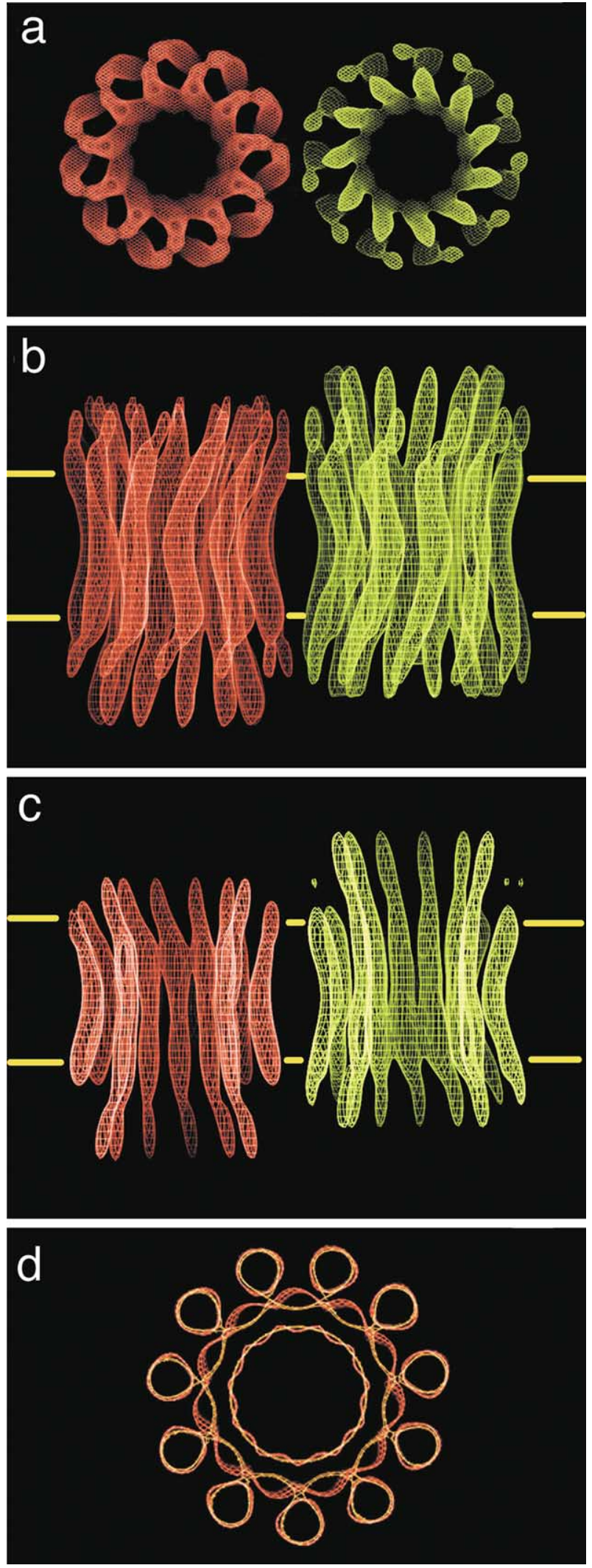

Figure 2. The 3-D density map of c-subunit rings after applying 11-fold non-crystallographic symmetry. The two rings in the unit cell are shown in red and green, respectively. (a) View normal to the membrane plane; the red ring is shown at two different contour levels, the lower one showing the loops. (b) Side view; the position of the membrane is indicated by yellow lines. (c) Cut-off
Analysis of these crystals by electron microscopy and correction of lattice distortions gave measurable amplitudes and phases to $4 \AA$ resolution. The 2-D crystals are of plane group p1 (Table 1) and contain two $c_{11}$ rings in the unit cell, facing in opposite directions. A projection map calculated to $4 \AA$ resolution (Figure 1 ) shows an inner and an outer ring of 11 densities each, representing the helices. A 3-D map was calculated from images of tilted crystals to $4 \AA$ resolution in-plane and $15 \AA$ vertically (Table 1). In the map, two concentric rings of 11 helices can be recognised. In the intact $\mathrm{F}_{0}$, the c-ring may be asymmetric because of interactions of a few subunits with the stalk formed by $\gamma$ and $\varepsilon^{4}$ and by lateral interaction with the a subunit, as well as differences in charge because of ion binding. ${ }^{28}$ However, all these interactions are missing from the isolated c-ring, so all subunits are equivalent and the rings have inherent 11-fold rotational symmetry. Imposing this non-crystallographic symmetry on the map greatly improved the quality. Symmetry was imposed independently on both rings in the asymmetric unit (Figure 2). Both symmetrised rings show the same features, which provides an independent check on the quality of the map.

The map (Figure 2) shows two rings in the unit cell of approximately cylindrical shape. The outer diameter is $\sim 45 \AA$ in the middle of the membrane and $\sim 50 \AA$ at the top and bottom, enabling crystal contacts between the interdigitating outer helices at this level. The ring has a height of about $70 \AA$ and encloses a central cavity with a diameter of $\sim 17 \AA$ with low density in the map. One side protrudes further from the membrane than the other by $\sim 10 \AA$, as was observed by AFM. ${ }^{5}$

The 2-D crystals contain a lipid bilayer into which the $\mathrm{c}_{11}$-rings are inserted in alternating up and down orientations. This enables us to deduce the position of the lipid bilayer relative to the c-ring surface: the centre plane of the 2-D crystal must correspond to the middle of the membrane. Assuming a thickness of the fatty acid tails of the lipid bilayer of $\sim 35 \AA,{ }^{29,30}$ the boundaries of the hydrophobic part of the membrane can be localised at equal distances above and below the central plane of the 2-D crystal (Figure 2). The yeast $F_{1} c_{10}$ crystals ${ }^{4}$ do not contain a lipid bilayer; therefore, the position of the $c_{10}$-rings in the membrane could not be deduced in that study.

The loops connecting membrane-spanning helices are rarely resolved in electron crystallographic maps. However, in the present map of the Ilyobacter c rings, the loops connecting the inner

side view at a higher contour level than in (b). Note the section of larger density on the inner helix on both rings, just after the loop region. (d) Section through the ring near the centre. Red, density as in (b) with all data to $4 \AA$ included. Yellow, a map with resolution cut-off at $6 \AA$. 


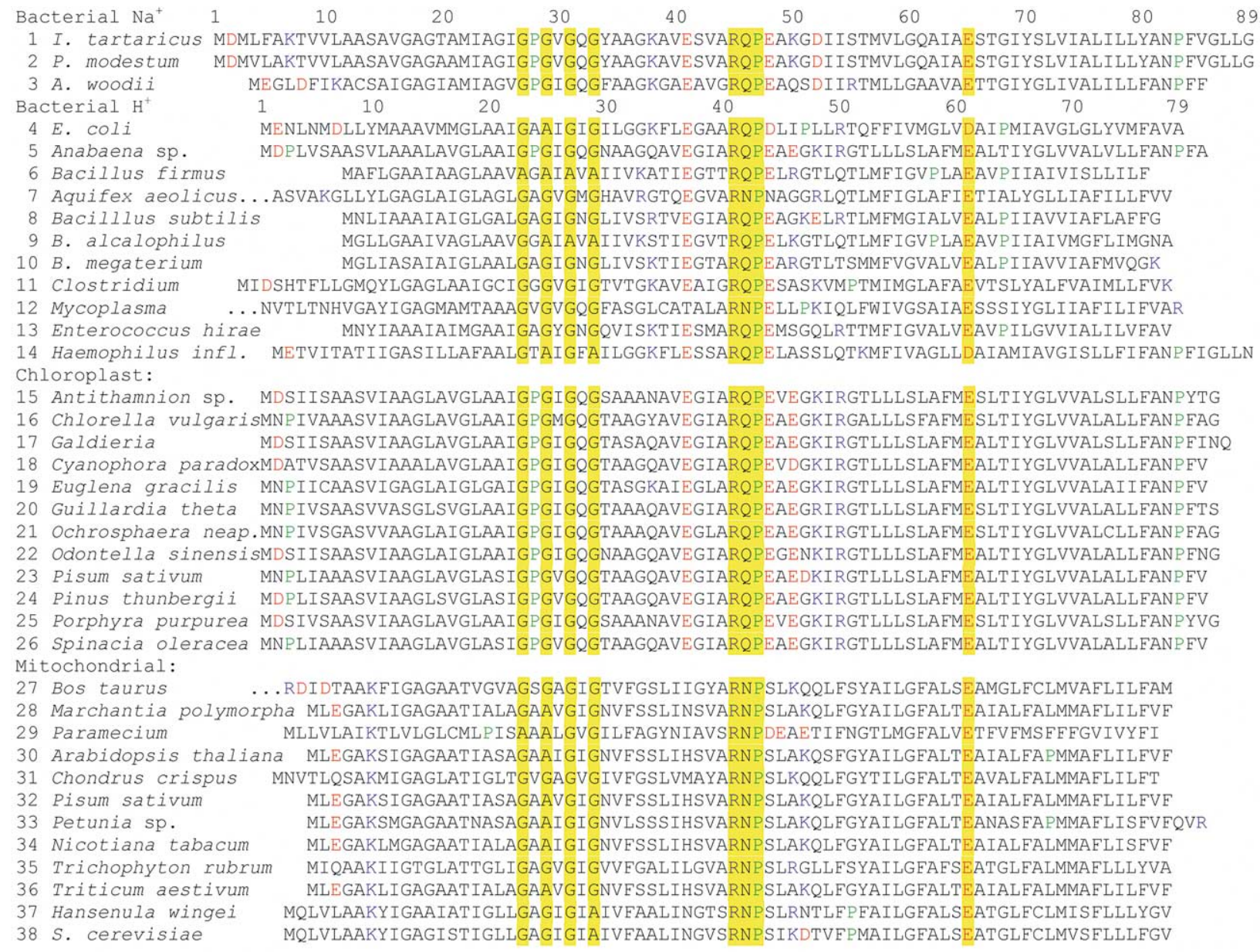

Figure 3. Alignment of c-subunit sequences for 38 species: $1-3$, bacterial $\mathrm{Na}^{+}$translocating; $4-14$, bacterial $\mathrm{H}^{+}$translocating; 15-26, chloroplast; 27-38, mitochondrial. Residue numbers are given for the I. tartaricus and the E. coli sequence. Basic residues are shown in blue, acidic residues in red, proline in green. Highly conserved positions are highlighted in yellow. Helical regions are indicated below the sequences.

and outer helices of each c subunit are clearly visible at low contour levels (Figure 2(a)), indicating that the loop is short and well ordered.

\section{Helix packing}

Electron crystallography can normally resolve transmembrane helices, especially when they are roughly perpendicular to the membrane. ${ }^{31-34}$ The I. tartaricus c-rings are unusual in this respect: at $7 \AA$ resolution, the inner ring of helices appears as a continuous, cylindrical wall of density (Figure 2(d)). The 11 inner-ring helices are resolved only when data to $4 \AA$ resolution in-plane are included, due to their unusually tight packing with a centreto-centre distance of $6.5 \mathrm{~A}$. The two helices of one c subunit are identified unambiguously in the map, as they are linked by the hairpin loop. The helices in the inner ring are straight, with an outward curve at both ends. In the central part, the helices are packed very tightly side-by-side. The tight helix packing leaves no room for side-chains, and therefore the residues at the helix interface must all be glycine. This enables us to assign the inner helices to the N-terminal half of the c subunit sequence. In Ilyobacter, a stretch of 26 residues starting at Ala12 contains nine Ala and eight Gly residues. Four of the glycine residues $(27,29,31$ and 33 in I.tartaricus) form a characteristic $x G x G x G x G x$ pattern (Figure 3). A helical wheel plot (Figure 4(a)) shows that the four conserved glycine residues fall on two opposite sides of the N-terminal helix, consistent with the tight side-byside packing of the inner helices. A comparison of sequences of $\mathrm{c}$ subunits from 38 widely different organisms shows that the $x G x G x G x G x$ pattern is highly conserved in all bacterial, chloroplast and mitochondrial ATP synthases. Glycine residues in this sequence are replaced by alanine only rarely (Figure 3). This suggests that the tight packing of the inner ring of helices is a feature common to the $c$ rings of all ATP synthases. The tight packing may be necessary to prevent the leakage of ions into the central cavity of the ring.

The helical wheel of Figure 4(a) shows that residues with large hydrophobic side-chains, primarily leucine and isoleucine, cluster on one side of the N-terminal helix (residues 23, 26, 30 

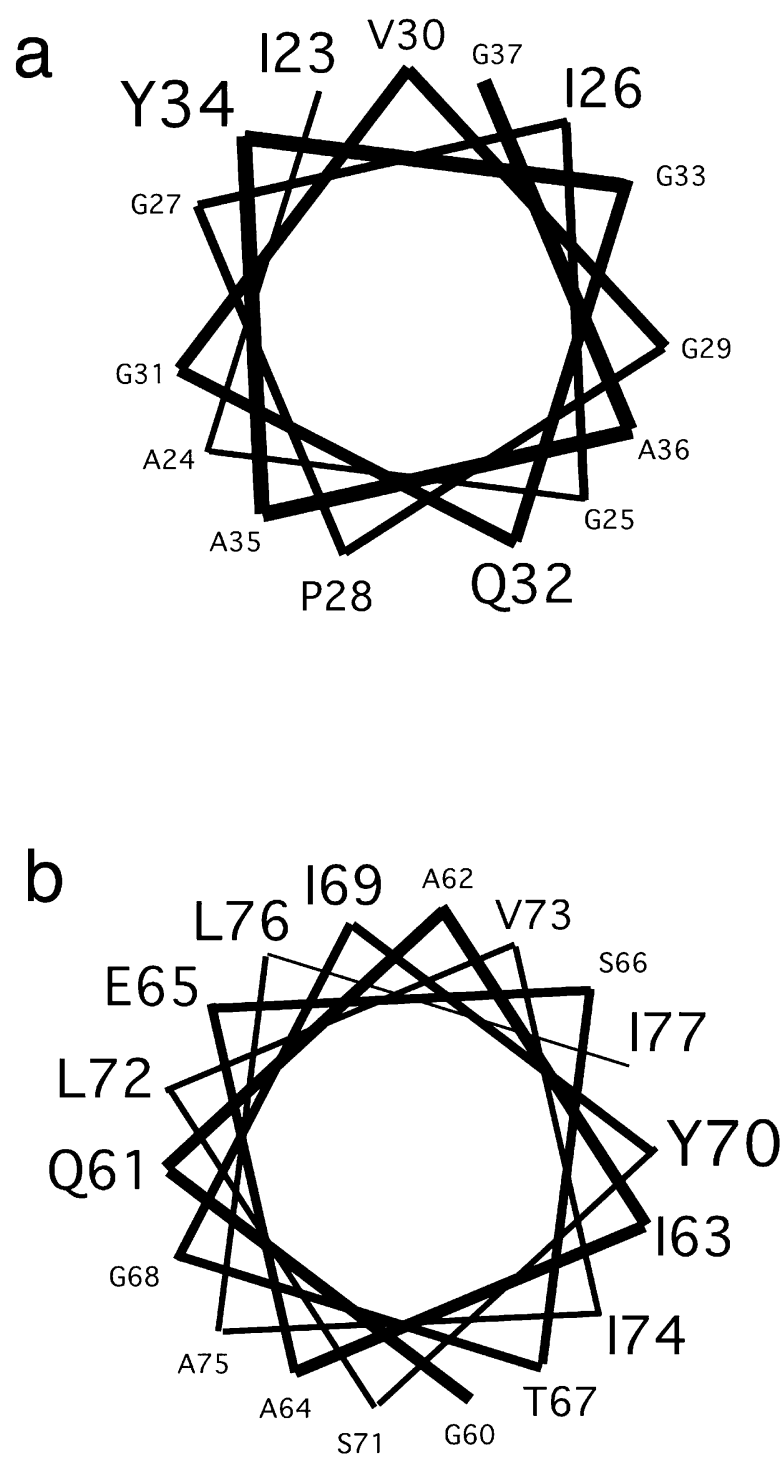

Figure 4. Helical wheel plots for the regions (a) Ile23Gly37 and (b) Gly60-Ile77. The size of the lettering indicates the size of the side-chain for the consensus residue at this position. The view is from the cytoplasmic side. The sequence shown is for the I. tartaricus c-subunit.

and 34), whereas there is only one large side-chain (Gln32 in I. tartaricus) on the opposite helix surface. Gln32 is part of the $\mathrm{Na}^{+}$-binding site in P. modestum, ${ }^{22}$ which shares a high level of homology with I. tartaricus (Figure 3), and therefore this side of the N-terminal helix has to face the Cterminal outer helix. Also on this helix face is a proline residue (Pro28) that is conserved completely in chloroplast ATP synthases and bacterial $\mathrm{Na}^{+}$-translocating ATP synthases (Figure 3). Pro28 has been suggested to play a role in the $\mathrm{Na}^{+}$-binding pocket. ${ }^{35}$ The cluster of bulky hydrophobic side-chains on the opposite side of the helix is thus facing the central cavity of the ring. At the periplasmic, N-terminal end, the inner helix turns slightly outward to accommodate the bulky first 11 residues, which in I. tartaricus contain no glycine and only a single alanine. This region of the structure varies in length in different species and is not highly conserved, but it always contains large and/or charged residues. At the cytoplasmic side, the inner helix also curves outwards.

Whereas the inner helix is curved only slightly, the outer, C-terminal helix has a more complex S-shape (Figure 2(c)). The first part of this helix runs at an angle of $\sim 25^{\circ}$ to the membrane normal. Near the level of Glu65 there is a tight bend of $\sim 30^{\circ}$. At this point the helix approaches the inner ring most closely, fitting into the groove between two inner helices. Whereas the E. coli c subunit has a proline residue at this position (Pro64), which would account for the change of helix direction, there is no such residue in I. tartaricus. The next segment of the outer helix runs along the groove between two N-terminal helices, turning slightly outward in order to accommodate the many bulky side-chains in this region (Figure 3). There is another, even tighter bend near the $C$ terminus, presumably at the level of Pro83, resulting in a change of helix direction by more than $45^{\circ}$. The density after this kink appears thinner than the rest of the helix. This may be due to the resolution anisotropy of the map, or to a greater flexibility and resulting disorder of the C-terminal end of the outer helix. In P. modestum and I. tartaricus, this part of the c subunits contains two residues (Phe84 and Leu87) that are involved in maintaining the $\mathrm{Na}^{+}$specificity of the ATP synthase; ${ }^{36}$ when mutated to the smaller residues Leu and Val, respectively, the enzyme transports only $\mathrm{H}^{+}$or $\mathrm{Li}^{+}$but not $\mathrm{Na}^{+}$.

\section{C- $\alpha$ model}

A $C$ - $\alpha$-carbon model for the $c-11$ ring was based on the following constraints. (i) The conserved Arg-Gln-Pro region is in the cytoplasmic loop connecting the $\mathrm{N}$ and C-terminal helices. ${ }^{37,38}$ (ii) The N-terminal helix is inside the ring. (iii) Gly27, Gly29, Gly31 and Gly33 face the neighbouring subunit in the ring. (iv) Gln32 faces the C-terminal helix. $^{22}$ (v) Glu65 and Ser66 face the N-terminal helix. ${ }^{22}$

In accordance with our map and other structural data, most of the structure was modelled as $\alpha$-helical, with the exception of the loop region. The model places Pro83 at a pronounced kink near the end of the outer helix, after which the density appears weaker. The stretch between Pro83 and the $\mathrm{C}$ terminus was modelled here as an $\alpha$-helix, which fits the length of this map segment. The model is shown in Figures 5 and 6.

\section{lon-binding pocket}

In the model (Figures 5 and 6), an ion-binding pocket is formed in the space defined by three helices, with Glu65 from one subunit, Ser66 from the neighbouring subunit, and Pro28 and Gln32 from the $\mathrm{N}$-terminal helix of the neighbouring 


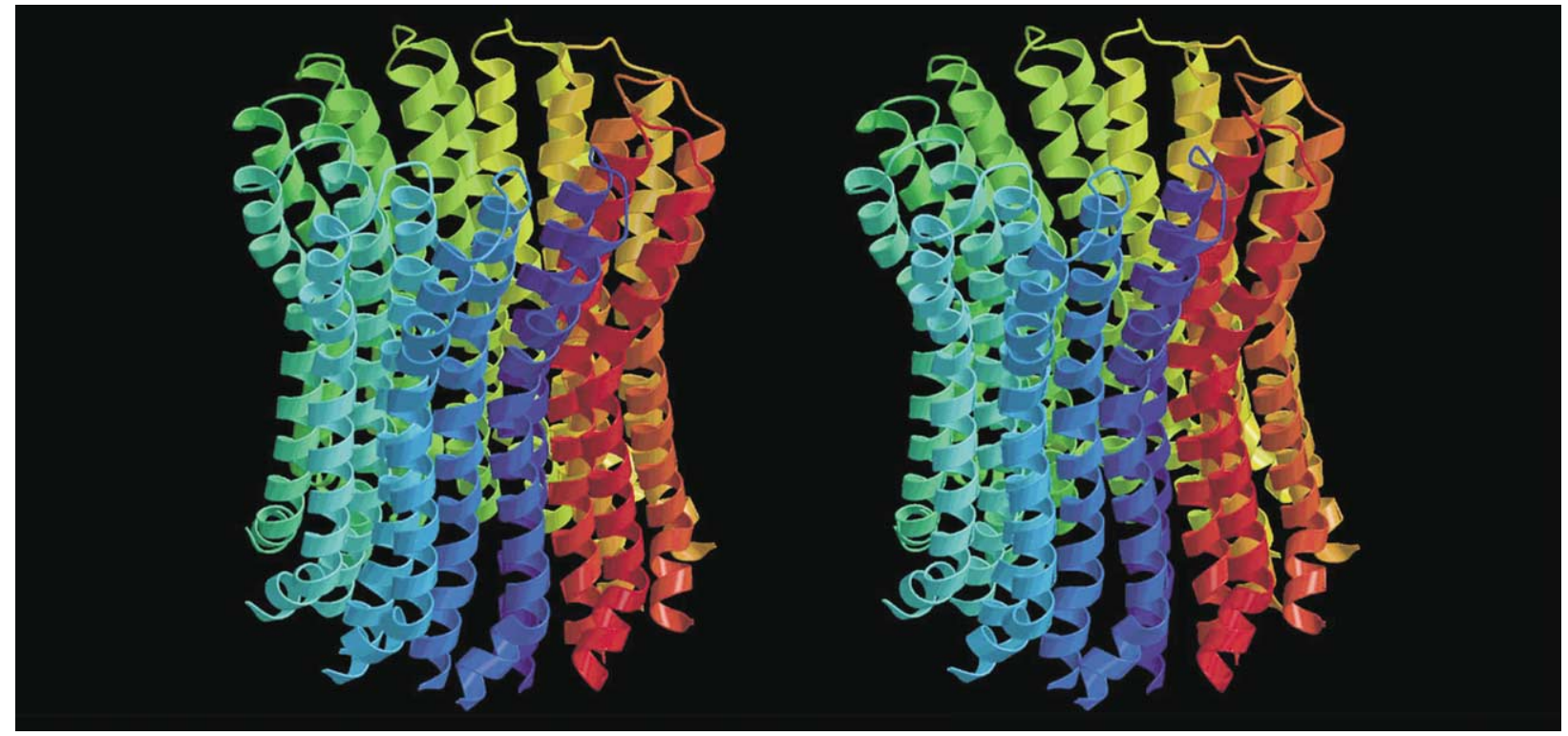

Figure 5. Stereo image of the $\mathrm{C}-\alpha$ model for the I. tartaricus c-11 ring. The image was created with Bobscript, ${ }^{49,50}$ a modification of MOLSCRIPT, ${ }^{51}$ and Raster3D. ${ }^{52}$

subunit. In the E. coli ac ac $_{12}$ model, ${ }^{28}$ which was not constrained by an experimentally derived map of an oligomeric ring, the N-terminal helices are further apart and the C-terminal helices are positioned radially outward (Figure $7(b))$, rather than fitting into the groove between two inner helices, as indicated by the I.tartaricus map. A $\mathrm{Li}^{+}$-binding site has been created by mutation of the E.coli c subunit, ${ }^{39}$ suggesting that the organisation of the E. coli $\mathrm{c}$ ring in the region of the ion-binding site is very similar to that of the I. tartaricus c ring. The model for the $\mathrm{H}^{+}$-transporting $c_{10}$ ring of yeast, which is based on a $4 \AA$ X-ray map (Figure $7(\mathrm{c})$ ), shows the same staggered helix arrangement as the I. tartaricus density, suggesting that this is a feature common to all c-subunit rings.

\section{Conserved residues in the $\mathrm{C}$-terminal helix}

Unlike the N-terminal helix, the C-terminal helix is rich in large hydrophobic side-chains (Leu, Ile, Phe and Tyr) (Figure 3). However, at several positions there is a conserved small residue: 60, 62, 64, 66, 68, 71 and 75 (Gly, Ala, Ala, Ser, Gly, Ser, Ala, respectively in I. tartaricus). With the exception of Ala62 and Ser66, these small residues cluster on one side on the helix, as can be seen in the helical wheel in Figure 4(b). In the model (Figure 6(b)), this helix face is oriented towards the outside of the ring. The absence of large sidechains in this region may be necessary for a close approach of the a subunit and its conserved arginine residue to the ion-binding site on the

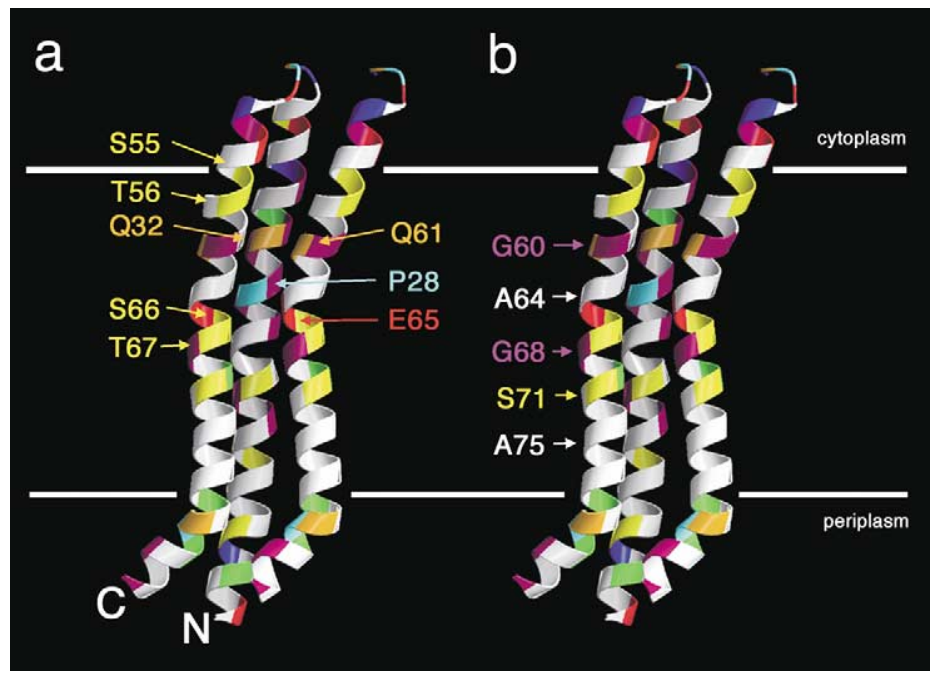

Figure 6. C- $\alpha$ model for the I. tartaricus $\mathrm{C}_{11}$ ring: close-up of the ion-binding site. For clarity, only one subunit is shown (with the $\mathrm{N}$ and $C$ terminus labelled) plus the neighbouring C-terminal helix. Colour code: red, negative; blue, positive; orange, polar (Asn, Gln); yellow, polar (Ser, Thr); green, aromatic; magenta, glycine; cyan, proline; grey, other hydrophobic residues. Membrane boundaries are indicated. (a) Residues involved in the binding site and the possible access route to the cytoplasm. (b) Conserved small residues on the outside of the helix. 


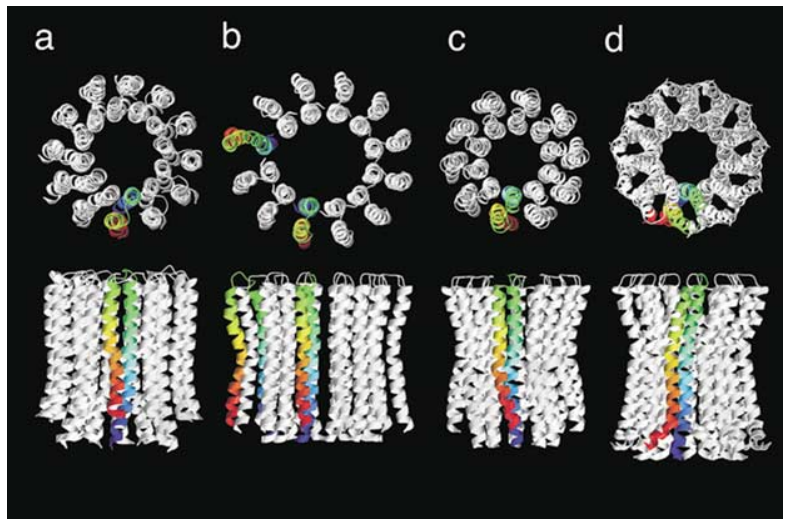

Figure 7. Comparison of c-ring models. (a) E. coli $\mathrm{c}_{12}$ model, ${ }^{43}$ PDB 1j7f. (b) The c-ring from E.coli $\mathrm{ac}_{12}$ model, ${ }^{28}$ from PDB 1c17. The coloured subunit on the left is deprotonated. (c) Yeast mitochondrial $\mathrm{c}_{10},{ }^{4}$ from PDB 1qo1. (d) I. tartaricus $c_{11}$ model, this study. Top: view normal to the membrane plane from the cytoplasmic side, below: side view. In every ring, one subunit is shown in colour from blue at the $\mathrm{N}$ terminus to red at the $\mathrm{C}$ terminus. In (a), also the deprotonated subunit (left) is shown in colour.

c-ring, to facilitate intersubunit rotation and ion translocation across the membrane.

\section{Phospholipids}

Analysis of the $c$ ring crystals by AFM showed that the central cavity is covered by a central plug on the side that protrudes more from the bilayer. The map shows that this is the periplasmic side (Figure 2). The plug can be removed by phospholipase and was therefore ascribed to phospholipid. ${ }^{40}$ In our model, hydrophobic residues line the central cavity on the periplasmic side, whereas it is lined by bulky, polar side-chains (Tyr34, Lys38 and Glu41) on the cytoplasmic side. This topography creates a favourable environment of the central cavity for the binding of phospholipids on the periplasmic side but not on the cytoplasmic side, in accord with the AFM data. Whether the phospholipid plug represents a structural feature of the ATP synthase complex in the membrane as well or forms during crystallisation is unknown.

\section{Ion-bridges}

The distribution of the charged residues in the c-subunit of I. tartaricus and that of its relatives P. modestum and Acetobacterium woodii ${ }^{35}$ provides a likely explanation for the extreme temperature stability of these c-rings, compared to that of other ATP synthases. ${ }^{26,41,42}$ In the $c$ subunit sequence of these three organisms, charged residues occur in pairs of positive and negative charges (Figure 3), except for Glu65, which defines the ion-binding site. The Arg45/Glu48 pair is thought to be involved in binding the $\varepsilon$ subunit, as has been reported for the equivalent residues Arg41/Asp44 in E. coli. ${ }^{38}$ The other ion pairs most probably form salt-bridges between neighbouring subunits. The map suggests that the N-terminal ion pair Asp2/ Lys7 occurs in an $\alpha$-helical context. Being five residues apart, they would be on opposite sides of the helix, and thus in favourable positions for forming an ion-bridge to the next c-subunit in the ring. The same is true for Lys50 and Asp52 at the outside of the loop, and Lys38 and Glu41 inside. Three intersubunit salt-bridges would indeed account for the extraordinary stability of the I. tartaricus and P. modestum c-rings. A single salt-bridge suggested for the corresponding region of the E. coli c-ring ${ }^{43}$ would be consistent with the lower temperature stability of this complex. The occurrence of these salt-bridges in I. tartaricus and P. modestum would explain why the c-rings disassemble at low $\mathrm{pH}$, where the protonation of the Asp or Glu sidechain would weaken the ion-bridges (unpublished results).

\section{Comparison with previous c-ring models}

The overall NMR structure of the monomeric E. coli c-subunit in organic solvent ${ }^{17,28}$ and our map agree in showing a straight $\mathrm{N}$-terminal helix and a kinked C-terminal helix. The I.tartaricus model is more similar to the structure determined at $\mathrm{pH} 5$, which has a protonated Asp61, than it is to the structure determined at $\mathrm{pH} 8,{ }^{28}$ where Asp61 would be deprotonated. In detail, however, the NMR structure of the E. coli c-subunit differs significantly from the I. tartaricus map in helix angles and curvature. These differences are most likely the result of interactions of the subunits within the oligomeric ring. Moreover, the structure of an isolated c-subunit in organic solvent may not reflect its native structure. Also, the sequences of E. coli and I.tartaricus are only $19 \%$ identical, which may give the proteins subtly different structures.

Several models have been proposed for the E. coli c-ring based on NMR models for the monomer in organic solvent (Figure $7(\mathrm{a})$ and (b)). ${ }^{28,43}$ In addition, the NMR model has been fit to the density of the yeast $c_{10}$ ring (Figure $\left.7(\mathrm{c})\right) .{ }^{4}$ The general features of these models are consistent: they all have an inner ring of N-terminal helices, and an outer ring of $\mathrm{C}$-terminal helices with a kink near the middle where the carboxylate group is located. The most conspicuous difference is the arrangement of C-terminal helices with respect to the inner ring. In all models except that of the E. coli $\mathrm{ac}_{12}$ complex, ${ }^{28}$ the C-terminal helices fit in a groove between two N-terminal helices. As discussed above, the more open arrangement of this model would result in a different binding pocket. As the yeast and I. tartaricus models are based on crystallographic structures of oligomeric rings, this arrangement seems to reflect the in vivo situation. Unlike Dmitriev et al., ${ }^{43}$ Rastogi \& Girvin ${ }^{28}$ constrained the interaction of the helices within 
a monomer to the NMR distances. It is likely that the helix interactions change upon oligomer formation, which would explain the difference between the models.

There are large differences between the arrangements of subunits within the ring of yeast and I. tartaricus in the angles of different parts of the helices (Figure 7(c) and (d)). The causes for this must be in the interactions between side-chains. Possibly, the proposed rings of ion-bridges in I. tartaricus force the helices into a different arrangement. The same forces may be responsible for the different numbers of subunits in the rings.

\section{Accessibility of the ion-binding site}

Monomers of the P. modestum c-subunit have been studied by NMR both in dodecylsulfate micelles ${ }^{19}$ and in the same organic solvent mixture that was used to analyse the NMR structure of the E. coli c subunit. ${ }^{20}$ Neither condition yielded a stable tertiary structure. Even the secondary structure was distinct in the different environments. Hence, any definite conclusions on the structure of subunit $c$ from a $\mathrm{Na}^{+}$-translocating $\mathrm{F}_{1} \mathrm{~F}_{0}$ ATP synthase had to await a crystal structure of the entire ring, as reported here. Of important functional significance is our finding that in the $c_{11}$ ring the $\mathrm{Na}^{+}$-binding site residues Gln32, Glu65 and Ser66 are located near the middle of the membrane (Figures 2 and 6). This location agrees perfectly with recent cross-linking data: a photoactivatable derivative of dicyclohexylcarbodiimide, which specifically modifies glutamate 65, was shown to cross-link only to the fatty acid sidechains of the lipid, indicating a location of Glu65 within the core of the membrane. ${ }^{25}$ Previously, a membrane-buried location of Glu65 was regarded unlikely on the basis of overwhelming biochemical evidence that $\mathrm{Na}^{+}$has access to the c subunit binding sites from the cytoplasmic surface entirely through the $c$ subunits itself and without participation of the a subunit. ${ }^{23,24}$ Possibly the most interesting aspect of the c-ring structure relates to these apparent contradictory observations: the spaces between the inner ring of helices and each two neighbouring outer helices represent putative $\mathrm{Na}^{+}$ access channels to the binding sites. Access of the site from the cytoplasmic surface by these channels is corroborated by a chain of polar and charged residues (Glu61, Thr56, Ser55, Asp52, Lys50) between the binding site and the cytoplasmic surface (Figure 6(a)), whereas the access of Glu65 from the periplasmic surface would be blocked by bulky hydrophobic side-chains.

\section{Materials and Methods}

ATP synthase was isolated from I.tartaricus cells as described. ${ }^{26}$ The $c_{11}$ oligomer was isolated from the ATP synthase and 2-D crystals were grown by a modification of the method described. ${ }^{5}$ Octylglucoside-solubilised protein with a final concentration of $1 \mathrm{mg} / \mathrm{ml}$ was mixed with palmitoyl-oleoyl phosphatidylcholine (POPC) at a lipid-to-protein ratio of $0.5 \%(\mathrm{w} / \mathrm{w})$ and a final concentration of $0.6 \%(\mathrm{w} / \mathrm{v}) \quad \beta$-octyl-glucoside. Micro-dialysis was performed in bent glass capillaries against $25 \mathrm{ml}$ of buffer $(10 \mathrm{mM}$ Tris- $\mathrm{HCl}(\mathrm{pH}$ 7.5), $200 \mathrm{mM} \mathrm{NaCl}, 3 \mathrm{mM} \mathrm{NaN}_{3}$ ) for 24 hours at $20^{\circ} \mathrm{C}$, then at $37^{\circ} \mathrm{C}$ for another 24 hours. To remove excess lipid, the harvested crystals were sedimented and the supernatant was exchanged against an equal volume of dialysis buffer containing no $\mathrm{NaCl}$.

Samples were prepared in $5 \%(\mathrm{w} / \mathrm{v})$ trehalose on molybdenum grids (Pacific Grid-Tech, San Diego, CA) by the back-injection method. ${ }^{44}$ Grids were examined in a JEOL 3000 SFF helium-cooled electron microscope at tilt angles of $0^{\circ}, 20^{\circ}, 30^{\circ}$ and $45^{\circ}$. Images were recorded by a spot-scanning procedure with $26 \times 32$ spots per image on Kodak SO-163 film at a magnification of $70,000 \times$. Films were digitised on a Zeiss SCAI scanner using a pixel size of $7 \mu \mathrm{m}$, corresponding to $1 \AA$ on the specimen. Images were corrected for lattice distortions and effects of the contrast transfer function using the MRC suite of programs. ${ }^{31,45,46}$ Data were merged to a resolution of $6 \AA$ for the tilted images and $4 \AA$ for the untilted images and a 3-D map was calculated with CCP4 programs. ${ }^{47}$ Amplitudes were scaled with a temperature factor $B=-200$ to compensate for a resolution-dependent degradation of amplitudes. Noncrystallographic symmetry was applied in real space using CCP4, independently for the two rings in the unit cell. The map was visualised in $\mathrm{O}^{48}$

\section{Acknowledgments}

We thank Cécile Breyton for discussions on noncrystallographic symmetry averaging. This work was supported by the DFG (Sonderforschungsbereich 472).

\section{References}

1. Abrahams, J. P., Leslie, A. G., Lutter, R. \& Walker, J. E. (1994). Structure at $2.8 \AA$ resolution of $F_{1}$ ATPase from bovine heart mitochondria. Nature, 370, 621-628.

2. Rodgers, A. J. W. \& Wilce, M. C. J. (2000). Structure of the $\gamma-\varepsilon$ complex of ATP synthase. Nature Struct. Biol. 7, 1051-1054.

3. Gibbons, C., Montgomery, M. G., Leslie, A. G. W. \& Walker, J. E. (2000). The structure of the central stalk in bovine $F_{1}$-ATPase at $2.4 \AA$ resolution. Nature Struct. Biol. 7, 1055-1061.

4. Stock, D., Leslie, A. G. \& Walker, J. E. (1999). Molecular architecture of the rotary motor in ATP synthase. Science, 286, 1700-1705.

5. Stahlberg, H., Müller, D. J., Suda, K., Fotiadis, D., Engel, A., Meier, T. et al. (2001). Bacterial $\mathrm{Na}^{+}$-ATP synthase has an undecameric rotor. EMBO Rep. 2, 229-233.

6. Seelert, H., Poetsch, A., Dencher, N. A., Engel, A., Stahlberg, H. \& Müller, D. J. (2000). Proton-powered turbine of a plant motor. Nature, 405, 418-419.

7. Birkenhäger, R., Hoppert, M., Deckers-Hebestreit, G., Mayer, F. \& Altendorf, K. (1995). The $\mathrm{F}_{0}$ complex of the Escherichia coli ATP synthase. Investigation by 
electron spectroscopic imaging and immunoelectron microscopy. Eur. J. Biochem. 230, 58-67.

8. Singh, S., Turina, P., Bustamante, C. J., Keller, D. J. \& Capaldi, R. (1996). Topographical structure of membrane-bound Escherichia coli $\mathrm{F}_{1} \mathrm{~F}_{0}$ ATP synthase in aqueous buffer. FEBS Letters, 397, 30-34.

9. Takeyasu, K., Omote, H., Nettikadan, S., Tokumasu, F., Iwamotu-Kihara, A. \& Futai, M. (1996). Molecular imaging of Escherichia coli $\mathrm{F}_{1} \mathrm{~F}_{0}$-ATPase in reconstituted membranes using atomic force microscopy. FEBS Letters, 392, 110-113.

10. Jiang, W. \& Fillingame, R. H. (1998). Interacting helical faces of subunits a and $c$ in the $\mathrm{F}_{1} \mathrm{~F}_{0}$ ATP synthase of Escherichia coli defined by disulfide cross-linking. Proc. Natl Acad. Sci. USA, 95, 6607-6612.

11. McLachlin, D. T., Bestard, J. A. \& Dunn, S. D. (1998). The $\mathrm{b}$ and $\mathrm{d}$ subunits of the Escherichia coli ATP synthase interact via residues in their C-terminal regions. J. Biol. Chem. 273, 15162-15168.

12. Wilkens, S. \& Capaldi, R. A. (1998). ATP synthase's second stalk comes into focus. Nature, 393, 29.

13. Noji, H., Yasuda, R., Yoshida, M. \& Kinosita, K. (1997). Direct observation of the rotation of $\mathrm{F}_{1}$-ATPase. Nature, 386, 299-302.

14. Sambongi, Y., Iko, Y., Tanabe, M., Omoto, H., Iwamoto-Kihara, A., Ueda, I. et al. (1999). Mechanical rotation of the c subunit oligomer in ATP synthase $\left(\mathrm{F}_{0} \mathrm{~F}_{1}\right)$ : direct observation. Science, 286, 1722-1724.

15. Pänke, O., Gumbiowski, K., Junge, W. \& Engelbrecht, S. (2000). F-ATPase: specific observation of the rotating c subunit oligomer of $\mathrm{EF}(0) \mathrm{EF}(1)$. FEBS Letters, 472, 34-38.

16. Boyer, P. D. (1993). The binding change mechanism for ATP synthase-some probabilities and possibilities. Biochim. Biophys. Acta, 1140, 215-250.

17. Girvin, M. E., Rastogi, V. K., Abilgaard, F., Markley, J. C. \& Fillingame, R. H. (1998). Solution structure of the transmembrane $\mathrm{H}^{+}$-transporting subunit $\mathrm{c}$ of the $\mathrm{F}_{1} \mathrm{~F}_{0}$ ATP synthase. Biochemistry, 37, 8817-8824.

18. Hoppe, J. (1984). Structure of the membraneembedded $\mathrm{F}_{0}$ part of $\mathrm{F}_{1} \mathrm{~F}_{0}$ ATP synthase from Escherichia coli as inferred from labeling with 3-(trifluoromethyl)-3-( $m-\left[{ }^{125} \mathrm{I}\right]$ iodophenyl)diazirine. Biochemistry, 23, 5610-5616.

19. Matthey, U., Kaim, G., Braun, D., Wütrich, K. \& Dimroth, P. (1999). NMR studies of subunit c of the ATP synthase from Propionigenium modestum in dodecylsulphate micelles. Eur. J. Biochem. 261, 459-467.

20. Matthey, U., Braun, D. \& Dimroth, P. (2002). NMR investigations of subunit $\mathrm{c}$ of the ATP synthase from Propionigenium modestum in chloroform:methanol: water (4:4:1). Eur. J. Biochem. 269, 1942-1946.

21. Dimroth, P., Wang, H., Gräbe, M. \& Oster, G. (1999). Energy transduction in the sodium F-ATPase of Propionigenium modestum. Proc. Natl Acad. Sci. USA, 96, 4924-4929.

22. Kaim, G., Wehrle, F., Gerike, U. \& Dimroth, P. (1997). Molecular basis for the coupling ion selectivity of $\mathrm{F}_{1} \mathrm{~F}_{0}$-ATPases: probing the liganding groups for $\mathrm{Na}^{+}$ and $\mathrm{Li}^{+}$in the $\mathrm{c}$ subunit of the ATP synthase from Propionigenium modestum. Biochemistry, 36, 9185-9194.

23. Kaim, G., Matthey, U. \& Dimroth, P. (1998). Mode of interaction of the single a subunit with the multimeric $c$ subunits during the translocation of the coupling ions by $\mathrm{F}_{1} \mathrm{~F}_{0}$ ATPases. EMBO J. 17, 688-695.
24. Kaim, G. \& Dimroth, P. (1998). Voltage-generated torque drives the motor of the ATP synthase. EMBO J. 17, 5887-5895.

25. von Ballmoos, C., Appoldt, Y., Brunner, J., Granier, T., Vasella, A. \& Dimroth, P. (2002). Membrane topography of the coupling ion binding site in $\mathrm{Na}^{+}$-translocating $\mathrm{F}_{1} \mathrm{~F}_{0}$ ATP synthase. J. Biol. Chem. 277, 3504-3510.

26. Neumann, S., Matthey, U., Kaim, G. \& Dimroth, P. (1998). Purification and properties of the $F_{1} F_{0}$ ATPase of Ilyobacter tartaricus, a sodium ion pump. J. Bacteriol. 180, 3312-3316.

27. Wehrle, F., Appoldt, Y., Kaim, G. \& Dimroth, P. (2002). Reconstitution of $F_{0}$ of the sodium ion translocating ATP synthase of Propionigenium modestum from its heterologously expressed and purified subunits. Eur. J. Biochem. 269, 2567-2573.

28. Rastogi, V. K. \& Girvin, M. E. (1999). Structural changes linked to proton translocation by subunit $\mathrm{c}$ of the ATP synthase. Nature, 402, 263-268.

29. Belrhali, H., Nollert, P., Royant, A., Menzel, C., Rosenbusch, J. P., Landau, E. M. \& Pebay-Peyroula, E. (1999). Protein, lipid and water organization in bacteriorhodopsin crystals: a molecular view of the purple membrane at $1.9 \AA$ resolution. Structure, 7, 909-917.

30. Luecke, H., Schobert, B., Richter, H. T., Cartailler, J. P. \& Lanyi, J. K. (1999). Structure of bacteriorhodopsin at 1.55 angstrom resolution. J. Mol. Biol. 291, 899-911.

31. Henderson, R., Baldwin, J. M., Ceska, T. A., Zemlin, F., Beckmann, E. \& Downing, K. H. (1990). A model for the structure of bacteriorhodopsin based on high resolution electron cryomicroscopy. J. Mol. Biol. 213, 899-929.

32. Kühlbrandt, W., Wang, D. N. \& Fujiyoshi, Y. (1994). Atomic model of plant light-harvesting complex by electron crystallography. Nature, 367, 614-621.

33. Walz, T., Hirai, T., Murata, K., Heymann, J. B., Mitsuoka, K., Fujiyoshi, Y. et al. (1997). The threedimensional structure of aquaporin-1. Nature, 387, 624-627.

34. Williams, K. A. (2000). Three-dimensional structure of the ion-coupled transport protein NhaA. Nature, 403, 112-115.

35. Rahlfs, S. \& Müller, V. (1997). Sequence of subunit c of the $\mathrm{Na}^{+}$-translocating $\mathrm{F}_{1} \mathrm{~F}_{0}$ ATPase of Acetobacterium woodii: proposal for determinants of $\mathrm{Na}^{+}$specificity as revealed by sequence comparisons. FEBS Letters, 404, 269-271.

36. Kaim, G. \& Dimroth, P. (1995). A double mutation in subunit $\mathrm{c}$ of the $\mathrm{Na}^{+}$-specific $\mathrm{F}_{1} \mathrm{~F}_{0}$-ATPase of Propionigenium modestum results in a switch from $\mathrm{Na}^{+}$to $\mathrm{H}^{+}$-coupled ATP synthesis in the Escherichia coli host cells. J. Mol. Biol. 253, 726-738.

37. Watts, S. D., Zhang, Y., Fillingame, R. H. \& Capaldi, R. A. (1995). The $\gamma$ subunit in the Escherichia coli ATP synthase complex $\left(\mathrm{ECF}_{1} \mathrm{~F}_{0}\right)$ extends through the stalk and contacts the $\mathrm{c}$ subunits of the $\mathrm{F}_{0}$ part. FEBS Letters, 368, 235-238.

38. Hermolin, J., Dmitriev, O. Y., Zhang, Y. \& Fillingame, R. H. (1999). Defining the domain of binding of $\mathrm{F}_{1}$ subunit $\varepsilon$ with the polar loop of $\mathrm{F}_{0}$ subunit $\mathrm{C}$ in the Escherichia coli ATP synthase. J. Biol. Chem. 274, 17011-17016.

39. Zhang, Y. \& Fillingame, R. H. (1995). Changing the ion binding specificity of the Escherichia coli $\mathrm{H}(+)$ transporting ATP synthase by directed mutagenesis of subunit c. J. Biol. Chem. 270, 87-93. 
40. Meier, T., Matthey, U., Henzen, F., Dimroth, P. \& Müller, D. J. (2001). The central plug in the reconstituted undecameric c cylinder of a bacterial ATP synthase consists of phospholipids. FEBS Letters, 505, 353-356.

41. Laubinger, W. \& Dimroth, P. (1987). Characterization of the $\mathrm{Na}^{+}$-stimulated ATPase of Propionigenium modestum as an enzyme of the $\mathrm{F}_{1} \mathrm{~F}_{0}$ type. Eur. J. Biochem. 168, 475-480.

42. Reidlinger, J. \& Müller, V. (1994). Purification of ATP synthase from Acetobacterium woodii and identification as a $\mathrm{Na}^{+}$-translocating $\mathrm{F}_{1} \mathrm{~F}_{0}$-type enzyme. Eur. J. Biochem. 223, 275-283.

43. Dmitriev, O., Jones, P. C. \& Fillingame, R. H. (1999). Structure of the subunit c oligomer in the Escherichia coli $\mathrm{F}_{0} \mathrm{~F}_{1}$ ATP synthase: model derived from solution structure of the monomer and cross-linking in the native enzyme. Proc. Natl Acad. Sci. USA, 96, $7785-7790$

44. Vonck, J. (2000). Parameters affecting specimen flatness of two-dimensional crystals for electron crystallography. Ultramicroscopy, 85, 123-129.

45. Henderson, R., Baldwin, J. M., Downing, K., Lepault, J. \& Zemlin, F. (1986). Structure of purple membrane from Halobacterium halobium: recording, measure- ment and evaluation of electron micrographs at $3.5 \AA$ A resolution. Ultramicroscopy, 19, 147-178.

46. Crowther, R. A., Henderson, R. \& Smith, J. M. (1996). MRC image processing programs. J. Struct. Biol. 116, 9-16.

47. Collaborative Computational Project No. 4 (1994). The CCP4 suite: programs for protein crystallography. Acta Crystallog. sect. D, 50, 760-763.

48. Jones, T. A. \& Kjeldgaard, M. (1993). O-The Manual, Uppsala University, Uppsala, Sweden.

49. Esnouf, R. M. (1997). An extensively modified version of MolScript that includes greatly enhanced coloring capabilities. J. Mol. Graph. Mod. 15, 132-134.

50. Esnouf, R. M. (1999). Further additions to MolScript version 1.4, including reading and contouring of electron-density maps. Acta Crystallog. sect. D, 55, 938-940.

51. Kraulis, P. J. (1991). MOLSCRIPT: a program to produce both detailed and schematic plots of protein structures. J. Appl. Crystallog. 24, 946-950.

52. Merritt, E. A. \& Bacon, D. J. (1997). Raster3D: photorealistic molecular graphics. Methods Enzymol. 277, 505-524.

53. Unger, V. M. \& Schertler, G. F. X. (1995). Low resolution structure of bovine rhodopsin determined by electron cryo-microscopy. Biophys. J. 68, 1776-1786.

Edited by W. Baumeister

(Received 2 April 2002; received in revised form 10 June 2002; accepted 11 June 2002) 